\title{
Marginally stable thick discs orbiting the Kerr-de Sitter black holes: the mass estimates
}

\author{
Petr Slaný and Zdeněk Stuchlík \\ Institute of Physics, Faculty of Philosophy and Science, Silesian University in Opava, \\ Bezručovo nám. 13, CZ-746 01 Opava, Czech Republic \\ email: zdenek.stuchlik@fpf.slu.cz, petr.slany@fpf.slu.cz
}

\begin{abstract}
Basic properties of equipotential surfaces in test perfect fluid tori with uniform distribution of the specific angular momentum orbiting KdS black holes are summarized. The central mass-densities of adiabatic non-relativistic tori, for which the approximation of test fluid is adequate, are given and compared with the typical densities of Giant Molecular Clouds.
\end{abstract}

Keywords. Accretion, accretion disks - black hole physics - cosmological parameters

Presence of a repulsive cosmological constant, $\Lambda>0$, changes the asymptotic structure of the black-hole backgrounds and implies strong consequences in the structure of thin and thick discs around Kerr-de Sitter (KdS) black holes, see the review of Stuchlík (2005).

KdS spacetimes are characterized by three parameters, $M, a, y$, representing the mass and spin of the black hole, and the dimensionless "cosmological parameter" defined in geometrical units $(c=G=1)$ as $y=\Lambda M^{2} / 3$. For simplicity, we put $M=1$ hereafter. Gravitational attraction of the black hole is just compensated by the cosmic repulsion at the "static radius", the only radius where the static geodesic observer resides: $r_{\mathrm{s}}=$ $y^{-1 / 3}$ in the Boyer-Lindquist coordinates. Stationary discs exist only in the spacetimes admitting stable circular geodesics. Beside the inner marginally stable (ms) and the inner marginally bound (mb) circular geodesics located near the black-hole horizon, there are the outer ms and mb circular geodesics near the static radius (Stuchlík \& Slaný (2004)).

Basic properties of geometrically thick discs are determined by the equilibrium configurations of test perfect fluid orbiting a black hole. Marginally stable thick discs are characterized by uniform distribution of the specific angular momentum, $\ell(r, \theta) \equiv-U_{\varphi} / U_{t}=$ const. Solving the Euler equation for the fluid with 4 -velocity $U^{\mu}=\left(U^{t}, 0,0, U^{\varphi}\right)$, see, e.g., AJS (1978), we obtain the relation for the potential $W$ in the form

$$
W(r, \theta)=\ln U_{t}(r, \theta)=\ln \left[\frac{\Sigma}{I^{2}} \cdot \frac{\Delta_{r} \Delta_{\theta} \sin ^{2} \theta}{\Delta_{\theta} \sin ^{2} \theta\left(r^{2}+a^{2}-a \ell\right)^{2}-\Delta_{r}\left(\ell-a \sin ^{2} \theta\right)^{2}}\right]^{1 / 2}
$$

$\Delta_{r}=r^{2}-2 r+a^{2}-y r^{2}\left(r^{2}+a^{2}\right), \quad \Delta_{\theta}=1+y a^{2} \cos ^{2} \theta, \quad I=1+y a^{2}, \quad \Sigma=r^{2}+a^{2} \cos ^{2} \theta$.

The boundary of any torus is given by any closed equipotential surface, however the last closed surface is critical enabling the outflows of matter from the disc through the cusp(s) due to the violation of hydrostatic equilibrium. We can distinguish three kinds of discs: accretion discs, marginally bound accretion discs and the excretion discs-completely new kind of toroidal structures from which the outflows into the outer space are only possible, see Fig. 1. Repulsive cosmological constant causes the existence of the outer cusp corresponding to the outer edge of the disc being located close to but bellow the static radius, and the strong collimation of open equipotential surfaces along the rotational axis 


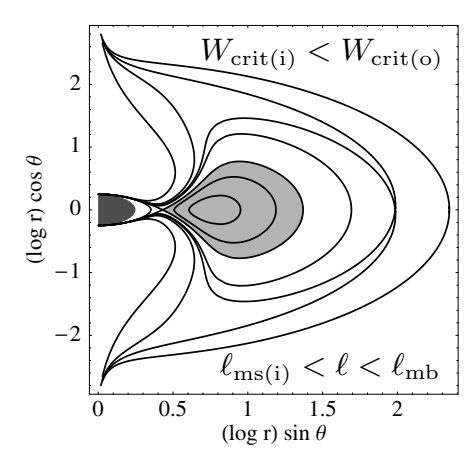

(a)

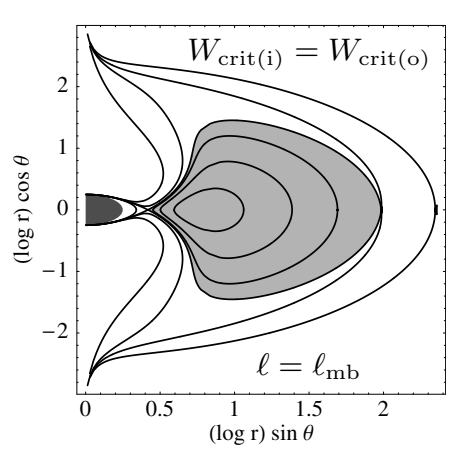

(b)

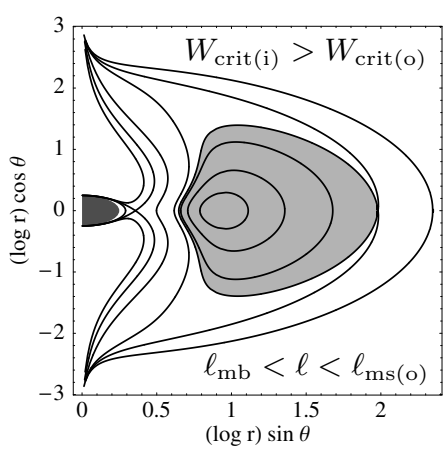

(c)

Figure 1. Typical behaviour of equipotential surfaces (meridional sections) in SdS and KdS black-hole spacetimes. Light gray region contains closed equipotential surfaces. The last closed surface is self-crossing in the cusp(s). Possible toroidal configurations correspond to: (a) accretion discs, (b) marginally bound accretion discs and (c) excretion discs.

indicating some role of $\Lambda>0$ for collimation of jets far away from the maternal galaxy. For the current value of the cosmological constant, $\Lambda_{0} \approx 1.3 \times 10^{-56} \mathrm{~cm}^{-2}$, the maximal dimensions of test disc-like structures around supermassive black holes are smaller but comparable with the dimensions of large galaxies, see dimensions of $r_{\mathrm{s}}$ in Table 1.

Relevance of test-fluid approximation can be tested by determining the central-mass density $\varrho_{\text {c }}$ of the torus for which the masses of the disc and central black hole are comparable. For an adiabatic nonrelativistic perfect fluid the total mass-energy of the torus is given by the relation bellow. The results are presented in Table 1. Comparing with the typical densities of Giant Molecular Clouds (GMC), $\varrho_{\mathrm{GMC}} \sim 10^{-18} \mathrm{~kg} \mathrm{~m}^{-3}$, we see that for diatomic GMC the limiting

Table 1. Mass parameter $(M)$, the static radius $\left(r_{\mathrm{s}}\right)$ and the central mass-density $(\varrho)$ of an adiabatic nonrelativistic perfect fluid torus corresponding to two values of the adiabatic index $\gamma=(5 / 3,7 / 5)$, for which $m_{\text {disc }} \approx M_{\mathrm{BH}}$, in $\operatorname{SdS}$ and $\operatorname{KdS}(a=0.9)$ black-hole spacetimes with $\Lambda_{0} \approx 1.3 \times 10^{-56} \mathrm{~cm}^{-2}$.

\begin{tabular}{cccccc}
\hline $\begin{array}{c}M \\
{\left[M_{\odot}\right]}\end{array}$ & $\begin{array}{c}r_{\mathrm{s}} \\
{[\mathrm{kpc}]}\end{array}$ & $\begin{array}{c}\varrho_{5 / 3} \\
{\left[\mathrm{~kg} \mathrm{~m}^{-3}\right]}\end{array}$ & $\begin{array}{c}\varrho_{7 / 5} \\
{\left[\mathrm{~kg} \mathrm{~m}^{-3}\right]}\end{array}$ & $\begin{array}{c}\varrho_{5 / 3} \\
{\left[\mathrm{~kg} \mathrm{~m}^{-3}\right]}\end{array}$ & $\begin{array}{c}\varrho_{7 / 5} \\
{\left[\mathrm{~kg} \mathrm{~m}^{-3}\right]}\end{array}$ \\
\hline $10^{6}$ & 10 & $10^{-22}$ & $10^{-13}$ & & \\
$10^{7}$ & 22 & $10^{-22}$ & $10^{-14}$ & $10^{-21}$ & $10^{-12}$ \\
$10^{8}$ & 50 & $10^{-22}$ & $10^{-15}$ & $10^{-22}$ & $10^{-13}$ \\
$10^{9}$ & 110 & $10^{-23}$ & $10^{-16}$ & $10^{-22}$ & $10^{-14}$ \\
\hline
\end{tabular}
$\varrho_{\mathrm{c}} \gg \varrho_{\mathrm{GMC}}$.

$$
m=2 \pi \varrho_{\mathrm{c}} \int_{\text {disc }}\left[\frac{1+\ell \Omega(r, \theta)}{1-\ell \Omega(r, \theta)}\right]\left[\frac{\exp \left\{W_{\text {in }}-W(r, \theta)\right\}-1}{\exp \left\{W_{\text {in }}-W_{\mathrm{c}}\right\}-1}\right]^{n}\left(r^{2}+a^{2} \cos ^{2} \theta\right) \sin \theta \mathrm{d} r \mathrm{~d} \theta
$$

This work has been done as a part of the research project MSM 4781305903.

\section{References}

Abramowicz, M. A., Jaroszyński, M. \& Sikora, M. 1978, A\&A, 63, 221

Slaný, P. \& Stuchlík, Z. 2005, Classical Quantum Gravity, 22, 3623

Stuchlík, Z. 2005, Mod. Phys. Lett. A, 20, 561

Stuchlík, Z. \& Slaný, P. 2004, Phys. Rev. D, 69, 064001 